\title{
Photon Can Be Described as the Normalized Mutual Energy Flow
}

\author{
Shuang-Ren Zhao \\ mutualenergy.org, London, Canada \\ Email:shrzhao@gmail.com
}

How to cite this paper: Zhao, S.-R. (2020) Photon Can Be Described as the Normalized Mutual Energy Flow. Journal of Modern Physics, 11, 668-682.

https://doi.org/10.4236/jmp.2020.115043

Received: March 28, 2020

Accepted: May 5, 2020

Published: May 8, 2020

Copyright $\odot 2020$ by author(s) and Scientific Research Publishing Inc. This work is licensed under the Creative Commons Attribution International License (CC BY 4.0).

http://creativecommons.org/licenses/by/4.0/

\begin{abstract}
Einstein guessed that the macroscopic electromagnetic wave is built by thousands of photons, however, no one has offered a theory about how the macroscopic electromagnetic wave is built from photons. A concrete theory about photons is needed to answer this question. Current theory for photons is Maxwell's equation which has the solution of waves, but it is difficult to describe the photon as a particle. There is the paradox problem of wave-particle duality. This article offers one solution to solve this problem by introducing the normalized mutual energy flow. The interaction of the retarded wave and advanced wave produce the mutual energy flow. The mutual energy flow satisfies the mutual energy flow theorem. The mutual energy flow theorem tells us that the energy that goes through each surface between the emitter and the absorber is all same. That means the mutual energy flow is different in comparison to the waves. The wave, for example, the retarded wave, its amplitude is decreased with the distance from the source to the point of the field. The mutual energy flow does not decrease. The author noticed this and claimed that the photon is the mutual energy flow. In this article the author updated this claim that the photon is the normalized mutual energy flow. Here the normalization of mutual energy flow will normalize the mutual energy flow to the energy of a photon, which is $\mathrm{E}=\mathrm{hf}$. $\mathrm{E}$ is the energy of the photon; $h$ is Planck constant; $f$ is the frequency of the light. This normalization is similar to the normalization in quantum mechanics. After this normalization the relation between an electromagnetic wave and photon as a particle becomes clear. This article will prove that the macroscopic wave of an electromagnetic field can be built by thousands of normalized mutual energy flows, which describes the photons. The mutual energy flow is an interaction of the retarded wave and the advanced wave. The retarded wave and the advanced wave satisfy the Maxwell equations. There are two additional waves which are the time-reversal waves which satisfy time-reversal Maxwell equations. The advanced wave and the two time-reversal waves are all real and physical electromagnetic fields. The time-reversal waves cancel all self-energy
\end{abstract}


flows of the retarded wave and advanced wave. Hence, the waves do not carry any energy, the energy is only transferred by the normalized mutual energy flows which are the photons. Hence, all energy is transferred by the photon instead of waves. This offers a solution to paradox of the duality of wave-particle.

\section{Keywords}

Advanced Wave, Retarded Wave, Time-Reversal Wave, Photon, Mutual Energy, Energy Flow, Electromagnetic Fields, Normalization, Wave-Particle Duality, Electron, Quantum

\section{Introduction}

\subsection{Mutual Energy Flow Can Be Applied to Normalize the Photon}

In quantum mechanics we can make normalization to an electron in the orbiter, which belongs to the stable solution of Schrödinger equation. We also can normalize the plane waves. However, we do not know how the normalization can be done for photon, or the electron in empty space. The reason is that we do not know how to describe the photon or electron in empty space. Someone perhaps will argue the photon can be described by Maxwell equations in the empty space, the electron can also be described as Schrödinger equation in empty space. That is true, but photon starts from an emitter and ends at an absorber. The energy is transferred from a point to another point without decrease. The solution of Maxwell equation is a wave which spreads to the whole space; hence, the amplitude of the wave decreases with the distance from the field point to the source. The wave solution and the particle have very big difference. Someone perhaps will argue that plane wave can describe the particle. But first, the charge cannot radiate plane wave, second the plane wave doesn't focus to two points in the place the photon is radiated and received. This is so called wave and particle duality paradox. Since this paradox, how the normalization of the photon in empty space occurs has not been solved yet. Similar situation happens also for electron in empty space. We need a wave package which can focus to point in both places of emitter and absorber; it is thick in the middle between the emitter and the absorber.

Recently this author has claimed that the photon is nothing else but the mutual energy flow [1]. The mutual energy flow is the interaction between the retarded wave sent from the source and the advanced wave sent from the absorber. This integration leads to an energy flow that starts from emitter ends at the absorber. The mutual energy flow theorem guarantees the energies go through any surfaces between the emitter and the absorber are all equal. Hence, the mutual energy flow can be a good role to do the normalization for photon. This article describes the details why and how to normalize the mutual energy flow and claim the photon is normalized mutual energy flow. 


\subsection{History Review about the Theory for Mutual Energy Flow}

In physics, Wheeler and Feynman introduced the absorber theory in 1945 and 1949 [2] [3], which claimed that the electric current can radiate advanced wave in the same time when it radiates the retarded wave. The absorbers send the advanced waves. This view of point has been extended to the transactional interpretation of quantum mechanics by Cramer around 1980 [4]. The theory about advanced wave of Wheeler and Feynman cited the earliest publications of action at a distance [5] [6] [7].

In electronic engineering, Welch has introduced his time-domain reciprocity theorem in 1960 [8]. In this theorem the transmitting antenna sends the retarded wave. The receiving antenna sends the advanced wave. Ramsey introduces his reciprocity theorem in 1963 [9]. This author introduced the mutual energy theorem in early of 1987 [10]. de Hoop introduced the cross-correlation reciprocity theorem in the end of 1987 [11]. All these theorems are same theorem and only have a difference in Fourier transform. Traditionally, when we speak about the reciprocity theorem, the two fields in the theorem do not need both to really exist. One field can be real and another can be virtual. In the reciprocity theorem of Welch, the retarded wave can be real, the advanced wave can be virtual, hence, the causality of physics has not been violated. However, when we speak these theorems as an energy theorem, it needs the two fields in the theorem to really exist. Hence, this author claimed this theorem is an energy theorem, that means the advanced wave must also exist in nature as a real substance. This author also applied the mutual energy theorem to Huygens principle, sphere wave and plane wave expansions in 1989 [12] [13].

Recently, this author reviewed the mutual energy theorem. Since, the search engine technology is much developed than before, when this time entering this field, this author found the cross-correlated reciprocity theorem of de Hoop [11]. From that, the author further found the time-domain reciprocity theorem of Welch [8]. Welch talked the concept of advanced wave, this author began to search the concept of advanced wave and noticed the publications of Wheeler and Feynman [2] [3], Cramer [4]. This author also noticed the publication of Stephenson [14], in which has a lot of interesting topic about advanced waves. After reading all these, this author believes the advanced wave really exists. After this, this author proved that the mutual energy theorem is an energy theorem [1] by proving it is a sub-theorem of Poynting theorem [14]. We know Poynting theorem is an energy theorem, hence, the mutual energy theorem is also an energy theorem. After these, this author found that the Poynting theorem conflicts with the energy conservation for $N$ charges in the empty space scenario. In order to solve this conflict, this author introduced the self-energy principle [1], which says corresponding to the retarded wave and advanced wave of the electromagnetic field, there are two time-reversal waves which cancel the self-energy flow of the retarded and advanced wave. Hence, the retarded wave and advanced wave do not carry any energy, they are probability waves instead of energy waves. 
Maxwell equations together with the self-energy principle can derive the mutual energy principle [1]. The mutual energy principle can also derive the Maxwell equations. However, the solutions of the mutual energy principle, are different with Maxwell equations. The Maxwell equations have two solutions, the retarded solution and the advanced solution, which exist independently. The solutions of the mutual energy principle are also the retarded solution and the advanced solution, but these two solutions exist together and must be synchronized together. The synchronized retarded wave and advanced wave can build the mutual energy flow which satisfies the mutual energy flow theorem. The mutual energy flow theorem is an extension of the mutual energy theorem. All these theories can be found in [1]. Details can also be found in [15] [16] [17].

\subsection{Normalized Mutual Energy Flow}

It should be clear in the mutual energy theorem, mutual energy flow theorem, mutual energy principle, the word "mutual" is only because the historical reason, this word can be dropped. It can be called as "energy theorem", "energy flow theorem", "energy principle". This is because the self-energy principle has told us the self-energy flow does not carry and transfer any energy, the energy is only carried and transferred by the mutual energy flow. Hence, the mutual energy flow is the exclusive energy flow. This energy flow has similar property of the photon. For example, the photon is substance that can transfer energy from a point (emitter) to another point (absorber). The photon energy that goes through each surface between the emitter and the absorber should be equal, which is the energy of the photon. This property of photon is just described by the mutual energy flow theorem. Hence, this author claimed that the photon is the mutual energy flow.

However, the mutual energy flow calculated by the classical electromagnetic field theory is decreased when the distance between the emitter and the absorber is increased. We know photon energy does not decrease with this distance and is a constant. Considering this, in this article, this author would like to normalize the mutual energy flow. After the normalization, the normalized mutual energy flow has exactly same properties as the photon.

It should be clear that, if the theory of mutual energy flow theorem, mutual energy principle, can still be put inside the frame of the Maxwell theory, the normalized mutual energy flow belongs to pure quantum effect and cannot be put inside the frame of Maxwell theory. However, this quantum effect has not been described by the current quantum mechanics theory. The preprint of this article can be found in ref [19].

\section{Mutual Energy Theorem and Mutual Energy Flow Theorem}

\subsection{Mutual Energy Theorem}

The Welch's reciprocity theorem can be written as, 


$$
\left(\xi_{1}(t), \tau_{2}(t)\right)=-\left(\tau_{1}(t), \xi_{2}(t)\right)
$$

where $t$ is time and,

$$
\begin{aligned}
& \xi_{i} \equiv\left[\boldsymbol{E}_{i}, \boldsymbol{H}_{i}\right], \quad i=1,2 \\
& \tau_{i} \equiv\left[\boldsymbol{J}_{i}, \boldsymbol{K}_{i}\right], \quad i=1,2
\end{aligned}
$$

$\boldsymbol{E}_{i}$ is electric field, $\boldsymbol{H}_{i}$ is magnetic field. $\boldsymbol{J}_{i}$ is electric current. $\boldsymbol{K}_{i}$ is magnetic current, which is 0 here. The two inner products are defined as,

$$
\begin{aligned}
& \left(\xi_{1}(t), \tau_{2}(t)\right) \equiv \int_{t=0}^{\infty} \mathrm{d} t \iiint_{V_{2}} \boldsymbol{E}_{1}(t) \cdot \boldsymbol{J}_{2}(t) \mathrm{d} V \\
& \left(\tau_{1}(t), \xi_{2}(t)\right) \equiv \int_{t=0}^{\infty} \mathrm{d} t \iiint_{V_{1}} \boldsymbol{J}_{1}(t) \cdot \boldsymbol{E}_{2}(t) \mathrm{d} V
\end{aligned}
$$

$\boldsymbol{J}_{1}$ is inside volume $V_{1} \cdot \boldsymbol{J}_{2}$ is inside $V_{2}$. The above formula. It should be clear that the above theorem can be easily extended to de Hoop's cross-correlation reciprocity theorem,

$$
\left(\xi_{1}(t+\tau), \tau_{2}(t)\right)=-\left(\tau_{1}(t+\tau), \xi_{2}(t)\right)
$$

The Fourier transform of the de Hoop's reciprocity theorem is the mutual energy theorem, the mutual energy theorem is in the Fourier domain, which still can be expressed as Equation (1). But with the meaning of inner product is in the Fourier transform domain, $\omega$ is angle frequency.

$$
\begin{gathered}
\left(\xi_{1}(\omega), \tau_{2}(\omega)\right)=-\left(\tau_{1}(\omega), \xi_{2}(\omega)\right) \\
\left(\tau_{1}(\omega), \xi_{2}(\omega)\right) \equiv \iiint_{V_{1}} \boldsymbol{J}_{1}(\omega) \cdot \boldsymbol{E}_{2}^{*}(\omega) \mathrm{d} V \\
\left(\xi_{1}(\omega), \tau_{2}(\omega)\right) \equiv \iiint_{V_{2}} \boldsymbol{E}_{1}(\omega) \cdot \boldsymbol{J}_{2}^{*}(\omega) \mathrm{d} V
\end{gathered}
$$

The above is Rumsey reciprocity theorem and also the mutual energy of this author. The above 4 theorems $(1,4,5)$ can be seen as same theorem in two different domain, time-domain and Fourier domain. It is clear that these theorems are reciprocity theorems, but this author claimed that it is also an energy theorem [10] and hence, called it as the mutual energy theorem. In the above theorem, this author usually assumed $\tau_{1}$ is the source, its field $\xi_{1}$ is the retarded field, $\tau_{2}$ is the sink, its field $\xi_{2}$ is the advanced field. This theorem tells us that the advanced wave $\xi_{2}$ sacked energy from the source $\tau_{1}$ which is equal to the energy that the retarded field $\xi_{1}$ offers to the sink $\tau_{2}$. In the formulas $(1,4$, $5)$ there is a negative sign, that is because $\tau_{1}$ is source, the source offers energy, hence, has a negative sign. Here the tradition is that the consumed energy is positive, hence, the energy offered by the source is negative. The right of the formula $(1,4,5)$ is the energy offered by the source $\tau_{1}$. The left of the formula $(1,3,5)$ is the consumed energy by the current of the sink $\tau_{2}$. The theorem tells us these two energies are equal.

\subsection{Mutual Energy Flow Theorem}

The above theorem has been extended as mutual energy flow theorem [1], 


$$
\left(\xi_{1}(t), \tau_{2}(t)\right)=\left(\xi_{1}(t), \xi_{2}(t)\right)=-\left(\tau_{1}(t), \xi_{2}(t)\right)
$$

The mutual energy flow is defined as

$$
\left(\xi_{1}(t), \xi_{2}(t)\right) \equiv \int_{t=0}^{\infty} \oiint_{\Gamma}\left(\boldsymbol{E}_{1}(t) \times \boldsymbol{H}_{2}(t)+\boldsymbol{E}_{2}(t) \times \boldsymbol{H}_{1}(t)\right) \cdot \hat{n} \mathrm{~d} \Gamma \mathrm{d} t
$$

$\Gamma$ is the arbitrary close surface or open surface which has extended to infinity between the source $\tau_{1}$ and the sink $\tau_{2}$, see Figure 1 .

The mutual energy flow theorem tells us, that the energy from the source to the sink is transferred through the mutual energy flow. The mutual energy flow is also a good inner product of the two fields, $\xi_{1}(t), \xi_{2}(t)$. The mutual energy flow theorem can also be converted to Fourier domain,

$$
\begin{gathered}
\left(\xi_{1}(\omega), \tau_{2}(\omega)\right)=\left(\xi_{1}(\omega), \xi_{2}(\omega)\right)=-\left(\tau_{1}(\omega), \xi_{2}(\omega)\right) \\
\left(\xi_{1}(\omega), \xi_{2}(\omega)\right) \equiv \oiint_{\Gamma}\left(\boldsymbol{E}_{1}(\omega) \times \boldsymbol{H}_{2}^{*}(\omega)+\boldsymbol{E}_{2}^{*}(\omega) \times \boldsymbol{H}_{1}(\omega)\right) \cdot \hat{n} \mathrm{~d} \Gamma
\end{gathered}
$$

The mutual energy flow theorem can be seen by Figure 1. In the Figure $\mathbf{a}$ is the source or emitter and $\mathbf{b}$ is the sink or absorber. The emitter can be seen as an atom with a transmitting antenna inside it. The absorber can be seen as an atom with a receiving antenna inside it. The source sends the retarded wave. The sink sends the advanced wave. The mutual energy flow is built by the retarded wave and advanced wave. The shape of the mutual energy flow is the overlap of retarded wave and the advanced wave which are thin in the two ends and thick in the middle. The mutual energy goes through any surfaces between $\mathbf{a}$ and $\mathbf{b}$ are equal. This is the mutual energy flow theorem. The mutual energy flow theorem further guarantees that the mutual energy theorem is an energy theorem.

\section{Normalized Mutual Energy Flow}

\subsection{Normalized Mutual Energy Flow}

This author has claimed that the photon is nothing else, but the mutual energy flow [1]. However, recently this author also noticed that the mutual energy flow calculated according to classical electromagnetic field theory decreases when the distance between the source and sink increases. This is clear, since the radiation fields decreases

$$
\begin{aligned}
& \xi_{1}\left(x_{2}\right) \propto \frac{1}{r} \\
& \xi_{2}\left(x_{1}\right) \propto \frac{1}{r} \\
& r \equiv\left\|x_{2}-x_{1}\right\|
\end{aligned}
$$

$x_{1}$ is the position of source. $\boldsymbol{x}_{2}$ is the position of sink. In other side we know the mutual energy flow should be the photon which has fixed energy $E=\hbar \omega$, where $\hbar$ is reduced Plank constant, $\omega$ is the angle frequency of the light. Hence, we require that,

$$
\left(\xi_{1}(t), \xi_{2}(t)\right)=\hbar \omega
$$




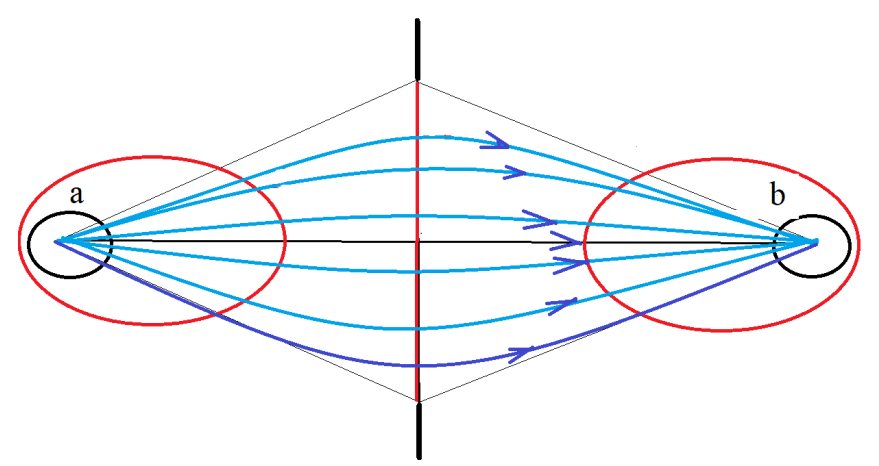

Figure 1. The mutual energy flow, which is started from source "a" and ended at sink "b". Source "a" is an emitter or transmitting antenna. Sink " $b$ " is absorber or a receiving antenna. Photon is the mutual energy flow. According to the mutual energy flow theorem, the mutual energy go through any of the surfaces between source "a" and sink " $b$ " are equal. The surfaces can be closed surface for example the sphere surface surrounding to the source "a" or the sink "b", or the infinite plane between "a" and "b".

In order to satisfy the above formula, we need to adjust the value of fields. We need to increase the fields according to the distance $r \equiv\left\|\boldsymbol{x}_{2}-\boldsymbol{x}_{1}\right\|$, that is,

$$
k r\left(\xi_{1}(t), \tau_{2}(t)\right)=k r\left(\xi_{1}(t), \xi_{2}(t)\right)=-k r\left(\tau_{1}(t), \xi_{2}(t)\right)=\hbar \omega
$$

$k$ is a constant which is not related the distance $r$. The above can be re-written as,

$$
\left(\xi_{1}^{p}(t), \tau_{2}^{p}(t)\right)=\left(\xi_{1}^{p}(t), \xi_{2}^{p}(t)\right)=-\left(\tau_{1}^{p}(t), \xi_{2}^{p}(t)\right)=\hbar \omega
$$

where,

$$
\begin{aligned}
& \xi_{i}^{p} \equiv \sqrt{k r} \xi_{i}(t), \quad i=1,2 \\
& \tau_{i}^{p} \equiv \sqrt{k r} \tau_{i}(t), \quad i=1,2
\end{aligned}
$$

$\xi_{i}^{p}$ is the electromagnetic fields of the photon, $\tau_{i}^{p}$ is the source of the photon. The field of photon is much stronger than the mutual energy flow calculated according to the classical electromagnetic fields, especially when the distance $r=\left\|\boldsymbol{x}_{1}-\boldsymbol{x}_{2}\right\|$ is very large. We can think that the energy is carried by the mutual energy flow of the classical electromagnetic field which is very small. However, this mutual energy flow builds an energy channel from emitter to the absorber. The energy channel can transfer the energy from emitter (source) to the absorber (sink). This energy channel can be applied repeatedly until the energy reach to the energy of one photon. That is the reason the photon field is stronger than the classical electromagnetic fields.

\subsection{Normalization the Wave Function in Quantum Mechanics}

In quantum mechanics there is also the normalization of the wave function. The square of amplitude of the wave function is normalized to 1 . This 1 is probability. The square of amplitude of wave function is normalized to 1 is similar to the normalized mutual energy flow to the energy of one photon. The difference between the two ways are only a constant. 
Because this author believes the wave is an energy-based wave instead of probability-based wave, the photon was normalized to the energy $\hbar \omega$. It should be clear that after the normalization, the field of photon $\xi_{i}^{p}$ is quite different with the classical electromagnetic field theory $\xi_{i}$. The photon field is normalized electromagnetic fields.

It should be clear, that in the current example the retarded field $\xi_{1}(\boldsymbol{x})=\left[\boldsymbol{E}_{1}, \boldsymbol{H}_{1}\right]$ is sent from the source a and hence, decreases according to $\left\|\boldsymbol{x}-\boldsymbol{x}_{1}\right\|$. The advanced field $\xi_{2}(\boldsymbol{x})=\left[\boldsymbol{E}_{2}, \boldsymbol{H}_{2}\right]$ is sent from the sink $\mathbf{b}$ and hence, decreases according to $\left\|x-x_{2}\right\|$. Even $\xi_{1}(x)$ and $\xi_{2}(x)$ decrease with the distance $\left\|x-x_{1}\right\|,\left\|x-x_{2}\right\|$, the mutual energy flow $\left(\xi_{2}(x), \xi_{1}(x)\right)$ is not changed at any surfaces see Figure 1 .

There is also another possibility that the advanced wave become the guidance wave of the retarded wave and the retarded wave become the guidance wave of the advanced wave. Because of the effect of guidance waves, the retarded wave and the advanced wave both do not decrease with the distance $\left\|\boldsymbol{x}-\boldsymbol{x}_{1}\right\|$ and $\left\|\boldsymbol{x}-\boldsymbol{x}_{2}\right\|$. However, inside the photon we cannot measure the field $\left[\boldsymbol{E}_{1}, \boldsymbol{H}_{1}\right]$ and $\left[\boldsymbol{E}_{2}, \boldsymbol{H}_{2}\right]$. This scenario has the same mutual energy flow as before. For calculating the mutual energy flow we still can use Equation (9).

\section{The Macroscopic Electromagnetic Field}

Here macroscopic electromagnetic field means the field produced by many photons together. This section we prove that the macroscopic electromagnetic field satisfy Maxwell equations of the retarded wave. We need a condition: all absorbers are uniformly distributed on the big sphere with its radius as infinite.

\subsection{Poynting Theorem Is Equivalent to Maxwell Equations}

It is known that the Maxwell equations,

$$
\begin{gathered}
\nabla \times \boldsymbol{E}=-\partial_{t} \boldsymbol{B} \\
\nabla \times \boldsymbol{H}=\boldsymbol{J}+\partial_{t} \boldsymbol{D} \\
\boldsymbol{B}=\mu_{0} \boldsymbol{H}, \boldsymbol{D}=\epsilon_{0} \boldsymbol{E}
\end{gathered}
$$

(where $\partial_{t}=\frac{\partial}{\partial t}$ ) is equivalent to the corresponding to Poynting theorem [18],

$$
-\oiint_{\Gamma} \boldsymbol{E} \times \boldsymbol{H}=\iiint_{V}\left(\boldsymbol{E} \cdot \boldsymbol{J}+\boldsymbol{E} \cdot \partial_{t} \boldsymbol{D}+\boldsymbol{H} \cdot \partial_{t} \boldsymbol{B}\right) \mathrm{d} V
$$

If we can prove the field of many photons satisfy Poynting theorem above we have proved that the macroscopic electromagnetic field satisfy Maxwell equations.

\subsection{The Self-Energy Principle and the Mutual Energy Principle}

Assume in the space there are $N$ charges. For the electromagnetic field of each charge, it satisfies Maxwell equations and hence, the Poynting theorem, 


$$
-\oiint_{\Gamma} \boldsymbol{E}_{i} \times \boldsymbol{H}_{i} \cdot \hat{n} \mathrm{~d} \Gamma=\iiint_{V}\left(\boldsymbol{E}_{i} \cdot \boldsymbol{J}_{i}+\boldsymbol{E}_{i} \cdot \partial_{t} \boldsymbol{D}_{i}+\boldsymbol{H}_{i} \cdot \partial_{t} \boldsymbol{B}_{i}\right) \mathrm{d} V
$$

Substitute the superposition principle

$$
\boldsymbol{E}=\sum_{i=1}^{N} \boldsymbol{E}_{i}, \quad \boldsymbol{H}=\sum_{i=1}^{N} \boldsymbol{H}_{i}
$$

to the Poynting theorem Equation (23) we obtain

$$
\begin{aligned}
& -\sum_{j=1}^{N} \sum_{i=1}^{N} \oiint_{\Gamma} \boldsymbol{E}_{j} \times \boldsymbol{H}_{i} \cdot \hat{n} \mathrm{~d} \Gamma \\
& =\sum_{j=1}^{N} \sum_{i=1}^{N} \iiint_{V}\left(\boldsymbol{E}_{j} \cdot \boldsymbol{J}_{i}+\boldsymbol{E}_{j} \cdot \partial_{t} \boldsymbol{D}_{i}+\boldsymbol{H}_{j} \cdot \partial_{t} \boldsymbol{B}_{i}\right) \mathrm{d} V
\end{aligned}
$$

Equation (24) is the energy of self-energy flow, we have introduced the self-energy principle [1] which tell us that self-energy flows do not carry any energy. The self-energy flow terms (which have $i=j$ ) have been all canceled by corresponding terms of the self-energy flows of the time-reversal waves. Hence, we can take away all self-energy flow terms from Equation (26), we obtain,

$$
\begin{aligned}
& -\sum_{j=1}^{N} \sum_{i=1, i \neq j}^{N} \oiint_{\Gamma} \boldsymbol{E}_{j} \times \boldsymbol{H}_{i} \cdot \hat{n} \mathrm{~d} \Gamma \\
& =\sum_{j=1}^{N} \sum_{i=1, i \neq j}^{N} \iiint_{V}\left(\boldsymbol{E}_{j} \cdot \boldsymbol{J}_{i}+\boldsymbol{E}_{j} \cdot \partial_{t} \boldsymbol{D}_{i}+\boldsymbol{H}_{j} \cdot \partial_{t} \boldsymbol{B}_{i}\right) \mathrm{d} V
\end{aligned}
$$

Equation (27) is the mutual energy formula, we have promoted it as the mutual energy principle, and claim it is real physical equation for photon [1]. From mutual energy principle, we can derive the Maxwell equations Equation $(20,21)$. However, the derived Maxwell equations must belong to the two groups, one is the retarded wave and another is the advanced wave. The retarded wave must synchronize with the advanced wave. The synchronized retarded wave and advanced wave can produce the mutual energy flow which satisfy the mutual energy flow theorem. Hence, the mutual energy principle is the foundation of the mutual energy flow theorem which describes the photon. In the follow section we will prove the macroscopic field i.e. the field of many photons that satisfy Maxwell equations by using the mutual energy principle. In this way, we have proved that the macroscopic wave can be built by thousands of photons together and proves Einstein's guesses.

\subsection{The Macroscopic Field of the Electromagnetic Field}

Equation (27) can be re-written as,

$$
\begin{aligned}
& -\sum_{j=1}^{N} \sum_{i=1}^{i<j} \oiint_{\Gamma}\left(\boldsymbol{E}_{j} \times \boldsymbol{H}_{i}+\boldsymbol{E}_{i} \times \boldsymbol{H}_{j}\right) \cdot \hat{n} \mathrm{~d} \Gamma \\
& =\sum_{j=1}^{N} \sum_{i=1}^{i<j} \iiint_{V}\left(\boldsymbol{E}_{j} \cdot \boldsymbol{J}_{i}+\boldsymbol{E}_{i} \cdot \boldsymbol{J}_{j}+\boldsymbol{E}_{j} \cdot \partial_{t} \boldsymbol{D}_{i}+\boldsymbol{E}_{i} \cdot \partial_{t} \boldsymbol{D}_{j}+\boldsymbol{H}_{j} \cdot \partial_{t} \boldsymbol{B}_{i}+\boldsymbol{H}_{i} \cdot \partial_{t} \boldsymbol{B}_{j}\right) \mathrm{d} V
\end{aligned}
$$

Considering among these charges there are $\mathrm{L}$ emitters and $\mathrm{M}$ absorbers. The retarded field of the emitter can only paired to an advanced field from absorbers, hence Equation (28) can be re-written as,

$$
\begin{aligned}
& -\sum_{j=1}^{L} \sum_{i=1}^{M} \oiint_{\Gamma}\left(\boldsymbol{E}_{j} \times \boldsymbol{H}_{i}+\boldsymbol{E}_{i} \times \boldsymbol{H}_{j}\right) \cdot \hat{n} \mathrm{~d} \Gamma \\
& =\sum_{j=1}^{L} \sum_{i=1}^{M} \iiint_{V}\left(\boldsymbol{E}_{j} \cdot \boldsymbol{J}_{i}+\boldsymbol{E}_{i} \cdot \boldsymbol{J}_{j}+\boldsymbol{E}_{j} \cdot \partial_{t} \boldsymbol{D}_{i}+\boldsymbol{E}_{i} \cdot \partial_{t} \boldsymbol{D}_{j}+\boldsymbol{H}_{j} \cdot \partial_{t} \boldsymbol{B}_{i}+\boldsymbol{H}_{i} \cdot \partial_{t} \boldsymbol{B}_{j}\right) \mathrm{d} V
\end{aligned}
$$


We consider a special situation, the source is at the origin point, there are infinite more absorbers are at the big sphere with its radius infinite large. Assume $L=1$, there is only one source (Emitter) which radiate the retarded wave. All other $\mathrm{M}$ charges are absorbers which are on the big sphere. In this special situation the above formula can be written as,

$$
\begin{aligned}
& -\sum_{i=1}^{M} \oiint_{\Gamma}\left(\boldsymbol{E}_{0} \times \boldsymbol{H}_{i}+\boldsymbol{E}_{i} \times \boldsymbol{H}_{0}\right) \cdot \hat{n} \mathrm{~d} \Gamma \\
& =\sum_{i=1}^{M} \iiint_{V}\left(\boldsymbol{E}_{0} \cdot \boldsymbol{J}_{i}+\boldsymbol{E}_{i} \cdot \boldsymbol{J}_{0}+\boldsymbol{E}_{0} \cdot \partial_{t} \boldsymbol{D}_{i}+\boldsymbol{E}_{i} \cdot \partial_{t} \boldsymbol{D}_{0}+\boldsymbol{H}_{0} \cdot \partial_{t} \boldsymbol{B}_{i}+\boldsymbol{H}_{i} \cdot \partial_{t} \boldsymbol{B}_{0}\right) \mathrm{d} V
\end{aligned}
$$

In the above, the retarded wave has the subscribe " 0 ". We have considered the advanced wave cannot build the mutual energy with advanced waves; hence, all absorber must build mutual energy with the only source. The above can be written as,

$$
\begin{aligned}
& -\oiint_{\Gamma}\left(\boldsymbol{E}_{0} \times \boldsymbol{H}_{a}+\boldsymbol{E}_{a} \times \boldsymbol{H}_{0}\right) \\
& =\iiint_{V}\left(\boldsymbol{E}_{0} \cdot \boldsymbol{J}_{a}+\boldsymbol{E}_{a} \cdot \boldsymbol{J}_{0}+\boldsymbol{E}_{0} \cdot \partial_{t} \boldsymbol{D}_{a}+\boldsymbol{E}_{a} \cdot \partial_{t} \boldsymbol{D}_{0}+\boldsymbol{H}_{0} \cdot \partial_{t} \boldsymbol{B}_{a}+\boldsymbol{H}_{a} \cdot \partial_{t} \boldsymbol{B}_{0}\right) \mathrm{d} V
\end{aligned}
$$

where $\boldsymbol{E}_{a}=\sum_{i=1}^{M} \boldsymbol{E}_{i}, \quad \boldsymbol{H}_{a}=\sum_{i=1}^{M} \boldsymbol{H}_{i}$ are superposed advanced wave. In the above formula, we can take the volume only includes the source. In this situation, the above formula become,

$$
\begin{aligned}
& -\oiint_{\Gamma}\left(\boldsymbol{E}_{0} \times \boldsymbol{H}_{a}+\boldsymbol{E}_{a} \times \boldsymbol{H}_{0}\right) \cdot \hat{n} \mathrm{~d} \Gamma \\
& =\iiint_{V}\left(\boldsymbol{E}_{a} \cdot \boldsymbol{J}_{0}+\boldsymbol{E}_{0} \cdot \partial_{t} \boldsymbol{D}_{a}+\boldsymbol{E}_{a} \cdot \partial_{t} \boldsymbol{D}_{0}+\boldsymbol{H}_{0} \cdot \partial_{t} \boldsymbol{B}_{a}+\boldsymbol{H}_{a} \cdot \partial_{t} \boldsymbol{B}_{0}\right) \mathrm{d} V
\end{aligned}
$$

$\boldsymbol{E}_{0} \cdot \boldsymbol{J}_{a}$ is disappear in the above formula, since $J_{a}$ are at the big sphere which is at outside of $V$. In this case the absorbers uniformly distribute on the big sphere, the advanced wave will exactly same as the retarded field radiate from the source which is at the origin. Hence, the above formula can be written as,

$$
\begin{aligned}
& -\oiint_{\Gamma}\left(\boldsymbol{E}_{0} \times \boldsymbol{H}_{0}+\boldsymbol{E}_{0} \times \boldsymbol{H}_{0}\right) \cdot \hat{n} \mathrm{~d} \Gamma \\
& =\iiint_{V}\left(\boldsymbol{E}_{0} \cdot \boldsymbol{J}_{0}+\boldsymbol{E}_{0} \cdot \partial_{t} \boldsymbol{D}_{0}+\boldsymbol{E}_{0} \cdot \partial_{t} \boldsymbol{D}_{0}+\boldsymbol{H}_{0} \cdot \partial_{t} \boldsymbol{B}_{0}+\boldsymbol{H}_{0} \cdot \partial_{t} \boldsymbol{B}_{0}\right) \mathrm{d} V
\end{aligned}
$$

or

$$
-2 \oiint_{\Gamma}\left(\boldsymbol{E}_{0} \times \boldsymbol{H}_{0}\right) \cdot \hat{n} \mathrm{~d} \Gamma=\iiint_{V}\left(\boldsymbol{E}_{0} \cdot \boldsymbol{J}_{0}+2\left(\boldsymbol{E}_{0} \cdot \partial_{t} \boldsymbol{D}_{0}+\boldsymbol{H}_{0} \cdot \partial_{t} \boldsymbol{B}_{0}\right)\right) \mathrm{d} V
$$

Assume the total electromagnetic field together of retarded field and the advanced field are

$$
\boldsymbol{E}=\boldsymbol{E}_{0}+\boldsymbol{E}_{a}, \quad \boldsymbol{H}=\boldsymbol{H}_{0}+\boldsymbol{H}_{a}
$$

We have

$$
\boldsymbol{E}=\boldsymbol{E}_{0}+\boldsymbol{E}_{0}=2 \boldsymbol{E}_{0}, \quad \boldsymbol{H}=\boldsymbol{H}_{0}+\boldsymbol{H}_{0}=2 \boldsymbol{H}_{0}
$$

Substitute it to Equation (34), we have,

$$
-\oiint_{\Gamma}(\boldsymbol{E} \times \boldsymbol{H}) \cdot \hat{n} \mathrm{~d} \Gamma=\iiint_{V}\left(\boldsymbol{E} \cdot \boldsymbol{J}_{0}+\boldsymbol{E} \cdot \partial_{t} \boldsymbol{D}+\boldsymbol{H} \cdot \partial_{t} \boldsymbol{B}\right) \mathrm{d} V
$$

This is the Poynting theorem of the total electromagnetic fields $\boldsymbol{E}, \boldsymbol{H} . \boldsymbol{J}_{0}$ is the current intensity of the source. Please notice we have assumed that the absorbers are uniformly distributed on the big sphere. In this situation, the 
total electromagnetic field which are sum of the retarded wave from the emitter and the advanced wave from the absorber satisfy the Poynting theorem. If this electromagnetic field satisfies Poynting theorem, it also satisfies the Maxwell equations. It seems that all the electromagnetic fields are started from the source. Hence, we have proved that the electromagnetic fields of a point source together with the fields of the absorbers at big sphere with infinite radius satisfy Maxwell equations. This field looks like the retarded field. Here it only looks like the retarded field. The fields $\boldsymbol{E}, \boldsymbol{H}$ actually are the summation of the retarded field radiate from the source and advanced wave radiate from the absorbers.

If the macroscopic electromagnetic fields of a point source with the field of the absorber satisfies Maxwell equations, we can extend this result to many point sources inside a region. It should be clear, here the total electromagnetic field are contributions of the retarded wave and the advanced wave, but it looks like as all the field are send as retarded wave from the emitter. Hence, for macroscopic wave, it still can be seen as retarded. Since the mutual energy flow are the energy flow of the photon, we have proved that the macroscopic wave can be built by many photons. This macroscopic wave can be seen as retarded wave even it is built from the mutual energy flows which are in turn built from retarded wave and advanced wave.

\subsection{The Effect of the Normalized Mutual Energy Flow}

In the above we have actually proved the total electromagnetic fields satisfy Maxwell equations. The total electromagnetic field has two parts one is the retarded field from the source, the other are advanced wave from the uniformly distributed absorbers on a big sphere. Normalized mutual energy flow is the photon energy, which is much stronger than the mutual energy flows calculated through classical electromagnetic field theory without the normalization. The number of the normalized mutual energy flows are also much less the mutual energy flows calculated from classical electromagnetic field theory. If the number of normalized mutual energy flows are enough more, they will have same effect as the mutual energy flows calculated through the classical electromagnetic field theory (which has much smaller values, but much larger in numbers).

If the absorbers could be seen as uniformly distribute at big sphere, the advanced waves of the total absorbers will have the same value as the retarded wave. Hence, the above proof in subsection 4.3 is also effective for the case of normalized electromagnetic fields, which is the field of photons.

\subsection{The Structure of the Macroscopic Electromagnetic Fields}

According to the discussion of this article, the structure of the electromagnetic fields can be shown in Figure 2. From these, we can see the macroscopic electromagnetic fields have a complicated structure. The macroscopic electromagnetic fields are built by infinite mutual energy flows. The effect of 


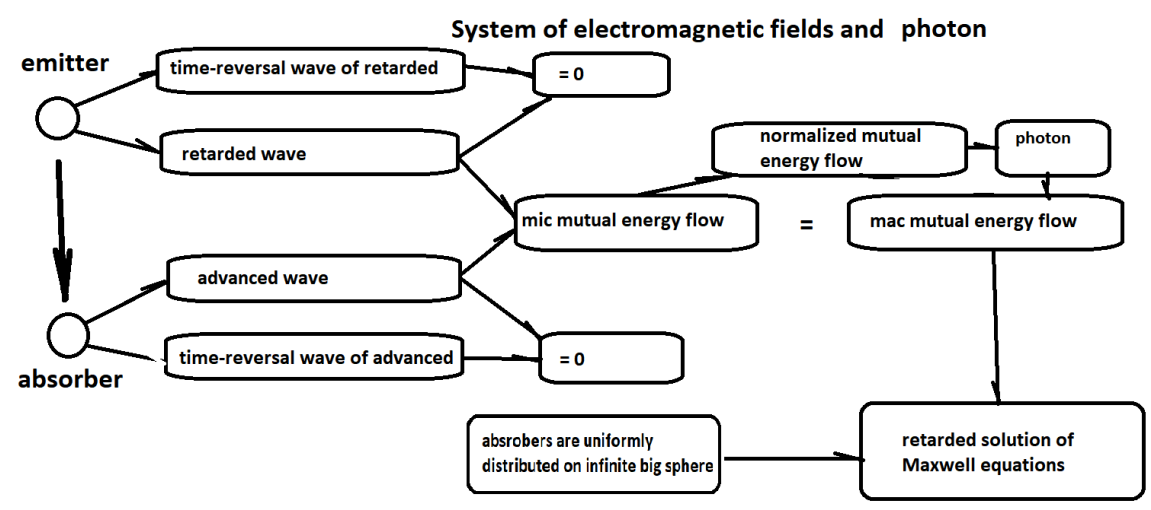

Figure 2. The structure of the electromagnetic field. In this figure the energy is sent from the emitter to the absorbers. Assume that the absorbers are uniformly distribute at big sphere with infinite radius. The emitter sends retarded wave and corresponding time-reversal wave. These two energy flows cancel. The absorber sends advanced wave and corresponding time-reversal wave. These two energy flows cancel. The retarded wave and the advanced wave interacted and produce the microscopic mutual energy flow. Normalized mutual microscopic energy flows are photons. Many photons have the same effect with many microscopic mutual energy flows. Macroscopic mutual energy flows have the same values as microscopic mutual energy flow. Macroscopic mutual energy flows can build the retarded solution of the Maxwell equations in case the absorber can be seen as uniformly distribute on the big sphere with infinite radius.

the mutual energy flow is same as infinite photons. It should be clear that the mutual energy flows are much smaller than the photon. However, when both mutual energy flows and photons have very big number, they are equivalent. In order to build the macroscopic wave to satisfy Maxwell equations, the absorbers have to be uniformly distributed on the big sphere with an infinite radius. Photons are the normalized mutual energy flows. After the normalization, the mutual energy flow has the energy of $E=\hbar \omega$. The mutual energy flows are built by the retarded wave sent from the emitter and the advanced wave sent from the absorber. The emitter also radiates the time-reversal wave which cancel the energy flow of the retarded wave. The absorber also radiates the time-reversal wave which cancel the energy flow of the advanced wave.

This figure tells us how macroscopic wave of electromagnetic field is built by photons which are normalized mutual energy flows. The mutual energy flow is the interaction of the retarded wave radiate from the source and the advanced wave radiate from the sink. The self-energy flow of the retarded wave and the advanced wave has been canceled by the corresponding time-reversal waves. Hence the waves, retarded and advanced wave, can be seen as probability wave, because they do not carry energy, if we consider the effect of the time-reversal waves. This means the microscopic waves are only look like probability wave, they are also real physic waves.

\section{In Case the Absorber Cannot Be Seen as Uniformly Distributed}

In order to test the above theory, we noticed that the above theory is only satis- 
fied in case the absorbers can be seen as uniformly distributed in big sphere with infinite radius. In case this condition cannot be satisfied, the result should be not obtained. That means the macroscopic wave of many photons should not satisfy Maxwell equations. This is true. For example, we assume there is a transmitting antenna radiate the wireless wave. We assume the environment can be seen as uniformly distributed absorbers. Hence, according to the above theory, the electromagnetic fields includes the retarded wave sent by the transmitting antenna and the advanced wave sent by the uniformly distributed absorbers together can be seen as retarded waves sent out from the transmitting antenna. Now we put a receiving antenna inside the above space. It is clear that when the receiving antenna is put in the system, closing to the receiving antenna, the absorbers cannot be seen as uniformly distributes on the big sphere. This means that closing to the receiving antenna, the electromagnetic field should not satisfy the Maxwell equations of the retarded wave. This is true. We know that for the receiving antenna, there are a concept of effective scattering section area. If effective scattering section area equals to the section area of the antenna, it tells us the field close to the antenna exactly satisfies the Poynting theorem. If the effective scattering section area larger than the section area of the receiving antenna, that means the Poynting theorem has not been exactly satisfied. We know from electronic engineering, for the receiving wire antenna, the effective scattering section area can be a hundred times larger than its section area. This means in this case the Poynting theorem cannot be satisfied. Since the Poynting theorem is equivalent to the two Maxwell equations Equation $(20,21)$, that means the Maxwell equations are also not satisfied.

In electronic engineering, the directivity dialog of receiving antenna cannot be calculated according to the theory of the retarded wave of Maxwell equations. Through the experiment we find the directivity dialog of the receiving antenna is same as the antenna used as the transmitting antenna. Hence engineers calculate the directivity dialog of the receiving antenna just using the same method as it is a transmitting antenna. This way it needs a proof that the directivity dialog of the receiving antenna is same as that of the transmitting antenna. Normally Lorentz reciprocity theorem is used for this task. It should be clear that the mutual energy flow theorem can also do this job. Since, in the directivity dialog is absolute value, and the phase information is omitted, and hence have the same value for the mutual energy theorem and Lorenz reciprocity theorem. Hence, the directivity dialog calculation for the receiving antenna is also because of the mutual energy theorem not Poynting theorem and also not Maxwell equations.

\section{Conclusions}

It is found that photon can be described by the normalized mutual energy flow. The mutual energy flow is the interaction of the retarded wave radiated from the source and the advanced wave radiated from the absorber. The normalization process is a process of physics which cannot be put in the frame of Maxwell theory. It is a pure quantum mechanics effect. 
After the normalization of the mutual energy flow, the mutual energy flow can be a good description of photon. The structure of the macroscopic electromagnetic field becomes clear; the macroscopic electromagnetic field is built by many photons. The effect of many photons is same as many mutual energy flows without the normalization. The mutual energy flow is built by the retarded wave and the advanced wave. The self-energy flow of the retarded wave and the advanced wave have canceled by the corresponding energy flow of the time-reversal waves of the retarded wave and the advanced wave.

With the theory of mutual energy flow and this normalization, the concept of collapse of the wave is not required. The wave and particle duality paradox have been solved.

\section{Conflicts of Interest}

The author declares no conflicts of interest regarding the publication of this paper.

\section{References}

[1] Zhao, S.R. (2017) American Journal of Modern Physics and Application, 4, 12-23.

[2] Wheeler, J.A. and Feynman, R.P. (1945) Reviews of Modern Physics, 17, 157. https://doi.org/10.1103/RevModPhys.17.157

[3] Wheeler, J.A. and Feynman, R.P. (1949) Reviews of Modern Physics, 21, 425. https://doi.org/10.1103/RevModPhys.21.425

[4] Schwarzschild, K. (1903) Nachrichten von der Gesellschaft der Wissenschaften zu Göttingen, 128, 132-141.

[5] Tetrode, H. (1922) Zeitschrift für Physik, 10, 137. https://doi.org/10.1007/BF01332555

[6] Fokker, A.D. (1929) Zeitschrift für Physik, 58, 386. https://doi.org/10.1007/BF01340389

[7] Cramer, J. (1986) Reviews of Modern Physics, 58, 647-688. https://doi.org/10.1103/RevModPhys.58.647

[8] Welch, W.J. (1960) IRE Transactions on Antennas and Propagation, 8, 68-73. https://doi.org/10.1109/TAP.1960.1144806

[9] Rumsey, V.H. (1963) IEEE Transactions on Antennas and Propagation, 11, 73-86. https://doi.org/10.1109/TAP.1963.1137982

[10] Zhao, S.R. (1987) Acta Electronica Sinica, 15, 88-93.

[11] de Hoop, A.T. (1987) Radio Science, 22, 1171-1178. https://doi.org/10.1029/RS022i007p01171

[12] Zhao, S.R. (1989) Journal of Electronics, 11, 204-208.

[13] Zhao, S.R. (1989) Journal of Electronics, 11, 73-77.

[14] Stephenson, L.M. (2000) Physics Essays, 13, 138.

[15] Zhao, S.R. (2020) Electromagnetic Field, Photon and Quantum with Advanced Wave and Time-Reversal Wave Based on Mutual Energy Principle and Self-Energy Principle (Volume I). https://hal.archives-ouvertes.fr/hal-02512141

[16] Zhao, S.R. (2020) Electromagnetic Field, Photon and Quantum with Advanced 
Wave and Time-Reversal Wave Based on Mutual Energy Principle and Self-Energy Principle (Volume II). https://hal.archives-ouvertes.fr/hal-02512153

[17] Zhao, S.R. (2020) Electromagnetic Field, Photon and Quantum with Advanced Wave and Time-Reversal Wave Based on Mutual Energy Principle and Self-Energy Principle (Volume III).

[18] Poynting, J.H. (1884) Philosophical Transactions of the Royal Society of London, 175, 343-361. https://doi.org/10.1098/rstl.1884.0016

[19] Zhao, S.R. (2019) Photon Is Interpreted by the Particleization/Normalization of the Mutual Energy Flow of the Electromagnetic Fields. https://vixra.org/abs/1910.0079 